\title{
Altered brain network centrality in patients with late monocular blindness: a resting-state fMRI study
}

Xin Huang ${ }^{1}$, Hai-Jun Li ${ }^{1}$, De-Chang Peng ${ }^{1}$, Lei Ye ${ }^{1}$, Qi-Chen Yang ${ }^{2}$, Yu-Lin Zhong ${ }^{1}$, Fu-Qing Zhou ${ }^{1}$, Yi Shao ${ }^{1}$

\author{
${ }^{1}$ Department of Ophthalmology, The First Affiliated Hospital of Nanchang University, \\ Nanchang, China \\ 2Department of Ophthalmology and Visual Sciences, The Chinese University \\ of Hong Kong, Hong Kong, China
}

Submitted: 21 December 2016

Accepted: 22 May 2017

Arch Med Sci 2019; 15 (5): 1301-1307

DOI: https://doi.org/10.5114/aoms.2019.87133

Copyright $\odot 2019$ Termedia \& Banach

\section{Abstract}

Introduction: The aim of the study was to investigate the underlying functional network brain activity changes in patients with late monocular blindness $(M B)$ and the relationship with their clinical features using the voxelwise degree centrality (DC) method.

Material and methods: A total of 32 patients with MB (25 males and $7 \mathrm{fe}$ males), and 32 healthy controls (HCs) (25 males and 7 females) closely matched in age, sex, and education, underwent resting-state functional magnetic resonance imaging scans. The DC method was used to assess local features of spontaneous brain activity. Correlation analysis was performed to explore the relationships between the observed mean $D C$ signal values of the different areas and clinical features in these patients.

Results: Compared with $\mathrm{HCs}, \mathrm{MB}$ patients had significantly lower $D C$ values in the bilateral cuneus/V1/V2, and significantly higher $D C$ values in the left inferior temporal gyrus and bilateral medial frontal gyrus. However, there was no relationship between the observed mean $D C$ values of the different brain areas and the behavioral performance.

Conclusions: Late monocular blindness involves brain function network dysfunction in many regions, which might indicate impairment of the visual cortex and other vision-related brain regions in the MBs.

Key words: monocular blindness, voxel-wise degree centrality, brainactivity changes.

\section{Introduction}

Blindness, a serious eye condition, is a worldwide health concern. The number of cases of blindness reached 32.4 million in 2010 globally [1]. Blindness is mainly characterized by the loss of light reaction from the surrounding environment. Blindness can be caused by a variety of eye diseases such as cataract [2], glaucoma [3] and optic neuritis [4]. Blindness can be roughly divided into reversible and irreversible blindness. Currently, surgery and drug therapy are effective for reversible blindness. Effective intraocular lens implantation can improve the visual quality of cataract-induced blindness [5]. Meanwhile, corticosteroids can be used to treat optic neuritis [6]. However, treatments for irreversible blindness such as optic atrophy are limited. The visual system includes the eyeball, visual pathways and visual cortex. Blindness may be accompanied by

\author{
Corresponding author: \\ Prof. Yi Shao \\ The First Affiliated \\ Hospital of \\ Nanchang University \\ 17 Yongwaizheng St \\ Donghu District \\ 330006 Nanchang, China \\ Phone: +86 13576955700 \\ E-mail: freebee99@163.com
}


dysfunction of the visual cortex and vision-related cortex.

Magnetic resonance imaging (MRI) has been successfully used to measure brain activities in many diseases $[7,8]$. It has been shown that blindness leads to abnormality of the visual cortex. Blind patients showed marked decreased gray matter volume (GMV) in the visual areas and sensorimotor areas compared with sighted subjects [9]. Meanwhile, other research revealed that early blindness showed decreased white matter volume (WMV) in the optic tract and optic radiation and marked reduced gray matter volume (GMV) in the visual cortex [10]. Blind subjects exhibited low fractional anisotropy in the ventral stream [11]. The abovementioned studies mainly focused on abnormalities of the brain anatomy and function in binocular blindness. However, alteration of the brain function network in monocular blindness is less well studied. Whether the monocular vision will lead to abnormal visual cortex remains unknown. Recently, voxel-wise degree centrality (DC) has been applied to evaluate the brain function network changes $[12,13]$. The $D C$ method measures the network architecture of functional connectivity within the human brain connectome at the voxel level $[14,15]$. It does not require the definition of regions of interest (ROIs). High DC values represent a node with numerous direct connections to other nodes. Thus, the $D C$ can detect the whole brain functional network [16]. The $D C$ method has been successfully used to evaluate pathological mechanisms of many diseases such as autism [17] and Parkinson's disease [18]. Thus, $D C$ is a reliable resting-state functional magnetic resonance technology.

Our study aimed to compare the whole brain function networks between the monocular blindness patients and sighted subjects using the voxel-wise $D C$ method, which might provide useful information for better understanding of the altered brain function networks in late monocular blindness.

\section{Material and methods}

\section{Subjects}

A total of 32 patients with MB (25 males and 7 females, all with right eye blindness) were recruited from the Ophthalmology Department of the First Affiliated Hospital of Nanchang University Hospital. Blindness was defined as best-corrected visual acuity (BCVA) of less than 0.05 (Snellen, 20/400) or a visual field no greater than $10^{\circ}$ around central fixation [19]. The diagnostic criteria of MBs were: 1) late stage of monocular blindness (eighteen patients with ocular trauma, fourteen patients with keratitis); 2) normal contralateral eye without any ocular diseases (cata- racts, glaucoma, optic neuritis, and retinal degeneration). The exclusion criteria were: 1) congenital blindness; 2) impaired contralateral eye vision; 3) a history of surgery in both eyes; 4) long-term medical treatment of blindness; 5) psychiatric disorders (depression, bipolar disorder, sleep disorder), and cerebral infarction diseases.

Thirty-two healthy controls ( 25 males, 7 females) with age-, sex-, and education statusmatched subjects were also recruited for this study. All healthy subjects met the following criteria: 1) no abnormalities in the brain parenchyma on cranial MRI; 2) no ocular diseases with uncorrected or corrected visual acuity $(\mathrm{VA})>1.0$; 3) no psychiatric disorders (depression, bipolar disorder, sleep disorder); and 4) be able to be scanned with MRI (e.g. no cardiac pacemaker or implanted metal devices). All research methods followed the Declaration of Helsinki and were approved by the principles of medical ethics of the First Affiliated Hospital of Nanchang University Hospital. All volunteers participated voluntarily and were informed of the purposes, methods, and potential risks before signing an informed consent form.

\section{MRI data acquisition}

All MRI data were collected on a Siemens Trio 3.0 T scanner. MRI scanning was performed on each subject. The MRI scanning parameters were as follows: repetition time $(T R)=2,000 \mathrm{~ms}$, echo time $(T E)=40 \mathrm{~ms}$, flip angle $=90^{\circ}$, slice thickness $/$ gap $=4.0 / 1 \mathrm{~mm}$, field of view $(F O V)=240 \mathrm{~mm} \times$ $240 \mathrm{~mm}$, in-plane resolution $=64 \times 64,30$ axial slices and 240 volumes.

\section{fMRI data preprocessing}

All the functional data were pre-filtered with MRIcro (www.MRIcro.com) and preprocessed using SPM8 (http://www.fil.ion.ucl.ac.uk/spm), DPARSFA (http://rfmri.org/DPARSF) and the Resting-state Data Analysis Toolkit (REST, http://www. restfmri.net). After removing the first 10 time points, the remaining 230 volumes were collected. Volumes with the $x, y$, or $z$ directions $>2^{\circ}$ were excluded from this study. More details were described in a previous study [20].

\section{Degree centrality}

Based on the individual voxel-wise functional network, $D C$ was calculated by counting the number of significant suprathreshold correlations (or the degree of the binarized adjacency matrix) for each subject. The voxel-wise DC map for each individual was converted into a z-score map using the following equation [20]: $Z_{i}=D C_{i}$ - mean $_{\text {all }}$ / $\operatorname{std}_{\text {all }}$, where $Z_{i}$ is $z$ score of the ith voxel, $D C_{i}$ is the $D C$ value of the ith voxel, mean ${ }_{\text {all }}$ is the mean $D C$ 
value of all voxels in brain mask, and $\mathrm{std}_{\text {all }}$ is the standard deviation of $D C$ values of all voxels in the brain mask.

\section{Statistical analysis}

For demographic and clinical measurements, the differences of clinical features between the MBs and HCs were calculated with independent-sample $t$-test using the SPSS 19.0 software (IBM, Armonk, NY).

For voxel-wise DC, the one-sample $t$-test was performed in each group to identify the spatial centrality distribution (hubs) of the whole brain functional network. Furthermore, we performed two-sample $t$-test using gender, age and education status as nuisance covariates within the default gray matter mask to assess between-group differences in the voxel-wise DC using REST V1.8. ( $p<0.05$ for multiple comparisons using Gaussian random field (GRF) theory 3, cluster-wise $p<0.05$ corrected was considered statistically significant.)

The relationship between the mean $D C$ values and the behavioral performance was calculated by correlation analysis.

\section{Clinical data analysis}

The cumulative clinical measurements, including the duration of the onset of monocular blindness and best-corrected VA, were recorded and analyzed in the study with the independent sample T test $(p<0.05$ was considered statistically significant).

\section{Results}

\section{Demographics and visual measurements}

The mean duration of the onset MBs was $22.63 \pm 7.60$ years. There were no obvious differences in weight $(p=0.482)$, age $(p=0.787)$, and best-corrected VA-Left $(p=0.438)$ between the patients with $M B$ and the HCs. However, there were marked differences in the best-corrected VARight $(p<0.001)$ between two groups. Details are presented in Table I.

\section{$D C$ differences}

Compared with HCs, MB patients had significantly decreased $D C$ values in the bilateral cuneus/V1/V2, and significantly increased $D C$ values in the left inferior temporal gyrus and bilateral medial frontal gyrus (Figures 1 A, B and Table II). Meanwhile, we showed the mean values of altered $D C$ between the MBs and HCs (Figure $1 \mathrm{C}$ ). In the MB group, there was no significant correlation between the observed mean $D C$ values of different brain areas and the behavioral performance $(p>0.05)$.

\section{Receiver operating characteristic curve}

We identified brain regions with different $D C$ values between the $M B$ and $H C$ groups, which might be used as markers to separate $M B$ from HCs. In our study, the area under the curve (AUC) was as follows: the bilateral cuneus/V1/V2 (0.790) (MBs < HCs) (Figure $2 \mathrm{~A}$ ); the left inferior temporal gyrus (0.651) and the bilateral medial frontal gyrus $(0.654)(\mathrm{MBs}>\mathrm{HCs})$ (B) (Figure $2 \mathrm{~B})$.

\section{Discussion}

The voxel-wise degree centrality (DC) method can be used to measure the whole-brain functional network at the voxel level. In our study, we found that the MB patients had significantly decreased $D C$ values in the bilateral cuneus/V1/V2, and significantly increased $D C$ values in the left inferior temporal gyrus and bilateral medial frontal gyrus.

\section{Analysis of the decreased $D C$ values in $M B$}

The visual cortex, located in the occipital lobe of the brain, is involved in the processing of visu-

Table I. Demographics and clinical measurements by group

\begin{tabular}{|lcccc|}
\hline Parameter & MB & HC & $t$ & $P$-value* \\
\hline Male/female & $25 / 7$ & $25 / 7$ & N/A & 0.99 \\
\hline Age [years] & $45.71 \pm 12.75$ & $44.85 \pm 12.64$ & 0.271 & 0.787 \\
\hline Weight [kg] & $58.90 \pm 6.36$ & $57.76 \pm 6.57$ & 0.708 & N/A \\
\hline Handedness & $32 \mathrm{R}$ & $32 \mathrm{R}$ & $\mathrm{N} / \mathrm{A}$ & $\mathrm{N} / \mathrm{A}$ \\
\hline Duration of MB [years] & $22.63 \pm 7.60$ & $\mathrm{~N} / \mathrm{A}$ & -23.596 & $<0.001$ \\
\hline Best-corrected VA-Right & $0.00 \pm 0.00$ & $1.21 \pm 0.29$ & -0.781 & 0.438 \\
\hline Best-corrected VA-Left & $1.18 \pm 0.16$ & $1.22 \pm 0.18$ & \\
\hline
\end{tabular}

*Independent $t$-tests comparing two groups ( $p<0.05$ indicates significant differences). MB - monocular blindness, $H C-$ healthy control, N/A - not applicable, VA - visual acuity. 


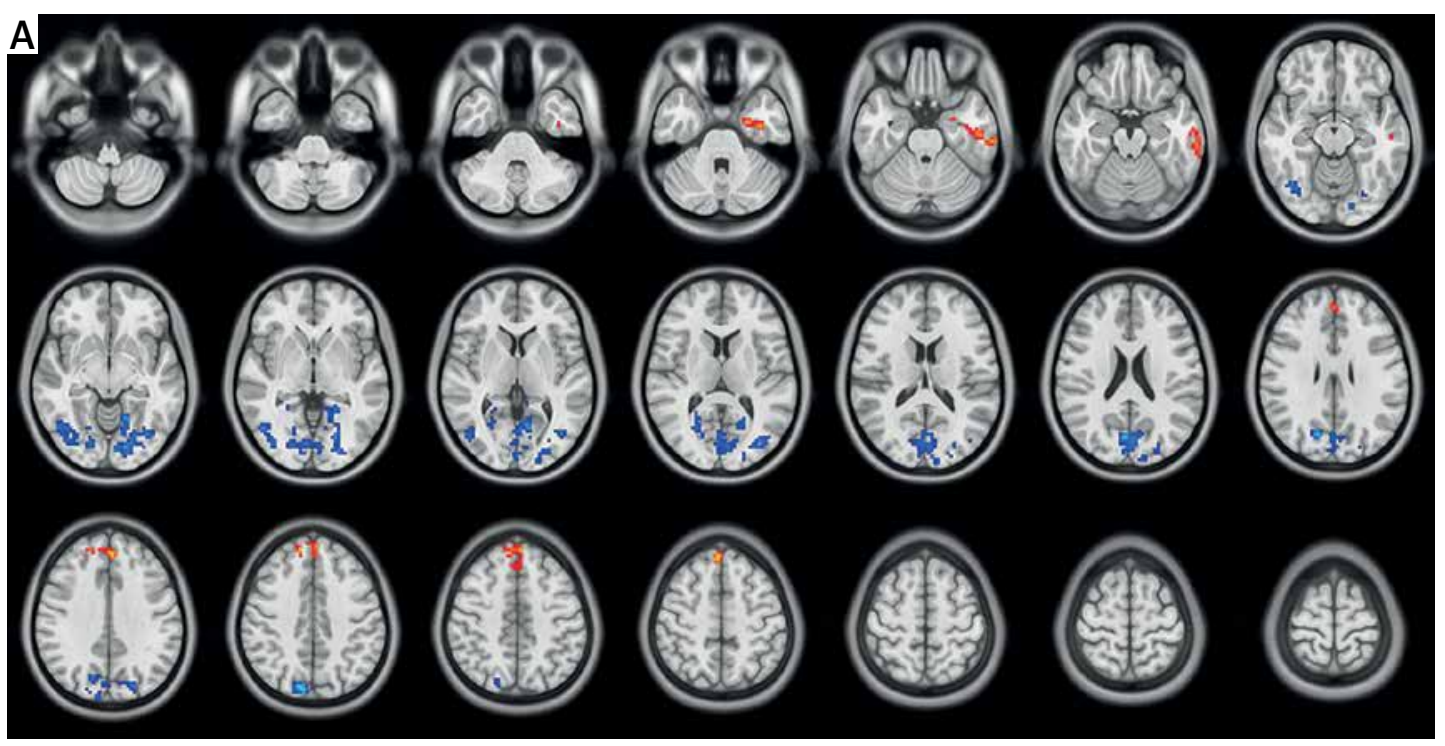

B
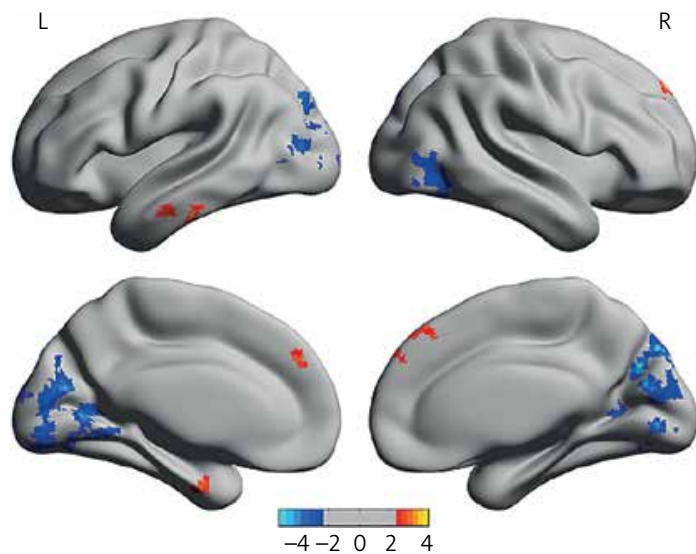

C

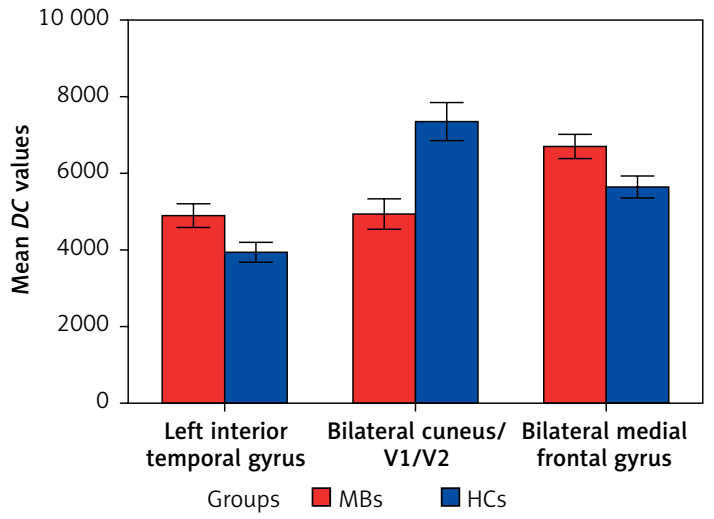

Figure 1. Voxel-wise comparison of DC in the MB and HC groups. A, B - Significant differences in DC were observed in the left inferior temporal gyrus, bilateral cuneus/V1/V2 and bilateral medial frontal gyrus; the red areas denote higher $D C$ values, and the blue denotes lower $D C$ values $(p<0.05$ for multiple comparisons using Gaussian random field (GRF) theory, $z>2.3$, cluster-wise $p<0.05$ corrected). $\mathbf{C}$ - The mean of altered $D C$ values between the MBs and $\mathrm{HCS}$

$D C$ - degree centrality, MB - monocular blindness, HC - healthy control, V1 - primary visual cortex, V2 - secondary visual cortex.

Table II. Brain areas with significantly different $D C$ values between groups. The statistical threshold was set at voxel level with $p<0.05$ for multiple comparisons using Gaussian random field (GRF) theory (cluster-wise $p<0.05$ corrected)

\begin{tabular}{|c|c|c|c|c|c|c|}
\hline \multirow{2}{*}{$\begin{array}{l}\text { Condition } \\
\text { Brain areas }\end{array}$} & \multirow[t]{2}{*}{ BA } & \multirow[t]{2}{*}{$T$-values } & \multirow{2}{*}{$\begin{array}{c}\text { Peak } \\
\text { voxels }\end{array}$} & \multicolumn{3}{|c|}{ MNI } \\
\hline & & & & $x$ & $y$ & $z$ \\
\hline Left inferior temporal gyrus & 20 & 3.788 & 125 & -36 & -9 & -30 \\
\hline Bilateral cuneus/V1/V2 & $18,19,7,30,31$ & -5.303 & 1107 & 15 & -72 & 30 \\
\hline Bilateral medial frontal gyrus & 8,9 & 4.448 & 153 & -3 & 45 & 30 \\
\hline
\end{tabular}

$D C$ - degree centrality, BA - Brodmann area, MB - monocular blindness, HCs - healthy controls, MNI - Montreal Neurological Institute, V1 - primary visual cortex, V2 - secondary visual cortex.

al information input from the lateral geniculate body. The primary visual cortex, also known as $\mathrm{V} 1$, is involved in the natural scene coding [21] and perception of color signals [22]. Additionally, V1 is responsible for modulation of spatial atten- tion [23]. After visual deprivation, retinal axons and optic nerve fibers slowly degenerate, and this leads to optic atrophy in the blind patients. Many previous studies demonstrated that blind patients showed impairment in the visual pathway [24,25]. 

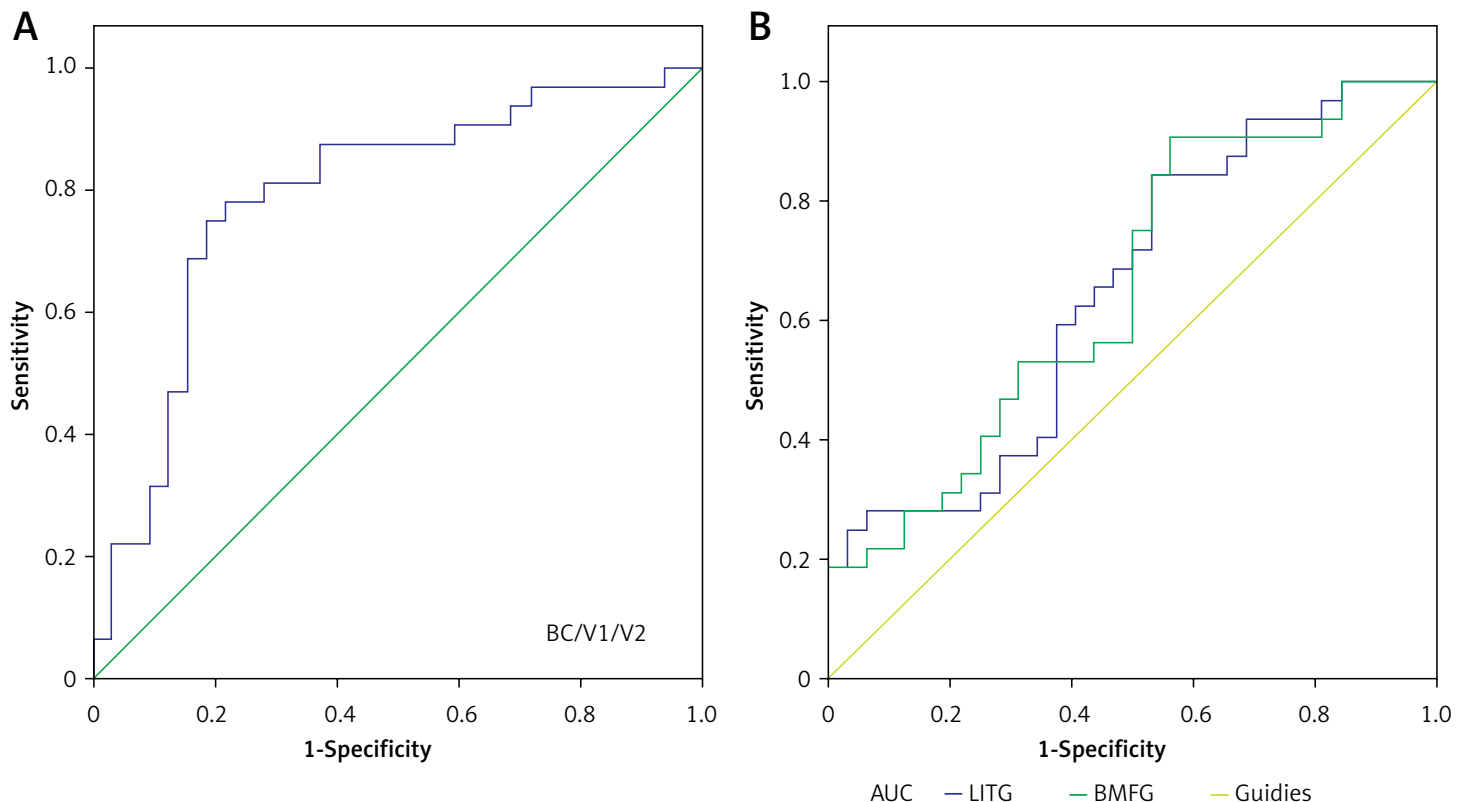

Figure 2. ROC curve analysis of the mean DC values for altered brain regions. The area under the ROC curve was 0.790 ( $p<0.001 ; 95 \% \mathrm{Cl}: 0.674-0.906)$ for the bilateral cuneus/V1/V2 (MBs < HCs) (A), and 0.651 ( $p=0.037$; $95 \% \mathrm{Cl}: 0.516-0.786)$ for the left inferior temporal gyrus, 0.654 ( $p=0.034 ; 95 \% \mathrm{Cl}: 0.520-0.789)$ for the bilateral medial frontal gyrus (MBs $>\mathrm{HCS})(\mathrm{B})$

$R O C$ - receiver operating characteristic, $D C$ - degree centrality, Cl-confidence interval, $V 1$ - primary visual cortex, V2 - secondary visual cortex.

Meanwhile, atrophy could lead to dysfunction of the visual cortex. A previous study demonstrated that early blindness showed lower levels of $\gamma$-amino acid butyric acid (GABA) in the occipital cortex [26]. Others reported that blind subjects exhibited decreased functional connectivity density in the primary visual cortex (V1) [27]. The abovementioned studies have shown that binocular blindness can lead to occipital visual cortex dysfunction. Furthermore, Zhong et al. demonstrated that macaque monkeys with monocular blindness showed reduced fractional anisotropy (FA) and increased mean diffusivity MD in the impaired optic tracts [28]. In our study, we also found significantly decreased $D C$ values in the bilateral $V 1$ in late right monocular blindness, which might reflect dysfunction of $\mathrm{V} 1$. We concluded that right monocular blindness might lead to impairment of bilateral V1.

The secondary visual cortex (V2) receives visual signals from $V 1$ and transduces the signals to a higher level of the visual cortex (V3, V4, V5). The $\mathrm{V} 2$ is also involved in the processing of color and motion [29]. In addition, it has also been suggested to regulate the illusory contour [30] and relative disparity [31]. Our study showed that the late right $M B$ had decreased $D C$ values in the bilateral V2, indicating dysfunction of V2. Dysfunction of the bilateral V1 might lead to impairment of the bilateral V2. Thus, we speculated that right MB might lead to defects of the bilateral V2.

The responses of neurons in the visual cortex (V1 and V2) were associated with stereo vision
[32]. Meanwhile, the disparity detectors were in cortical visual areas V1, V2, V3, V3A, VP, and MT (V5), which play an important role in stereopsis vision [33]. The normal binocular field is very important for binocular vision function. The MB patients had defects in binocular vision, which might lead to decreased $D C$ values in the bilateral V1/V2. Thus, we speculated that single vision might lead impairment of the bilateral V1/V2. The cuneus located in the occipital lobe of the brain is involved in visual processing. The occipital lobe including the visual cortex is the visual processing center. A previous study reported that the cuneus modifies visual information to extrastriate cortices via the primary visual cortex V1 [34]. In our study, we found that the MB showed significantly decreased $D C$ in the bilateral cuneus. These results suggest that late right $M B$ might lead to impairment of the bilateral visual cortex.

\section{Analysis of the increased $D C$ values in $M B$}

The inferior temporal gyrus (IT) located in the anterior of the temporal lobe controls the natural scenes coding along with the ventral visual pathway [35]. Moreover, the IT is responsible for visual shape categorization [36] and visual imagery [37]. A previous study reported that blind subjects showed lower functional connectivity between the left superior and middle temporal gyrus compared with sighted subjects [38]. In our study, we found that the MBs showed significantly increased $D C$ 
values in the left inferior temporal gyrus, which might reflect the enhanced activities of the IT. We speculated that the increased functional network in the IT might reflect the compensation of the monocular vision loss in the right MBs.

The medial frontal gyrus (MFG) located in the middle of the frontal gyrus is involved in empathy [39]. Meanwhile, the MFG is responsible for negative emotions [40]. Dysfunction of the MFG is associated with many diseases such as schizophrenia [41] and adjustment disorder [42]. In our study, we found that MB patients had significantly increased $D C$ values in the bilateral medial frontal gyrus, which might reflect the dysfunction of emotion in MBs. These results suggest that MB might be associated with emotion disorders.

In our study, we selected patients with right eye blindness, while left eye blindness patients were not included. The results might be more accurate if both eye patients were involved in the study. In a future study, we would compare the $D C$ differences between left and right eye blindness.

In conclusion, our results showed an abnormal brain function network in many brain regions, which might reflect the abnormalities of the visual cortex and other vision-related brain regions in MB.

\section{Conflict of interest}

The authors declare no conflict of interest.

\section{References}

1. Stevens GA, White RA, Flaxman SR, et al.; Vision Loss Expert Group. Global prevalence of vision impairment and blindness: magnitude and temporal trends, 19902010.Ophthalmology 2013; 120: 2377-84.

2. Thomas BJ, Sanders DS, Oliva MS, et al. Blindness, cataract surgery and mortality in Ethiopia. $\mathrm{Br} J$ Ophthalmol 2016; 100: 1157-62.

3. Pleet A, Sulewski M, Salowe RJ, et al. Risk factors associated with progression to blindness from primary open-angle glaucoma in an african-american population. Ophthalmic Epidemiol 2016; 23: 248-56.

4. Şahin S, Kola M, Cansu A, Kul S, İmamoğlu Hi. Chiasmal optic neuritis may be secondary to pneumococcal meningitis: a child with acute monocular vision loss. Neurol Sci 2016; 37: 139-41.

5. Li EY, Liu Y, Zhan X, et al. Prevalence of blindness and outcomes of cataract surgery in Hainan Province in South China. Ophthalmology 2013; 120: 2176-83.

6. Gal RL, Vedula SS, Beck R. Corticosteroids for treating optic neuritis. Cochrane Database Syst Rev 2015; 8: CD001430.

7. Liu H, Wang X. Correlation of iron deposition and change of gliocyte metabolism in the basal ganglia region evaluated using magnetic resonance imaging techniques: an in vivo study. Arch Med Sci 2016; 12: 163-71.

8. Bekiesińska-Figatowska M, Helwich E, Rutkowska M, Stankiewicz J, Terczyńska I. Magnetic resonance imaging of neonates in the magnetic resonance compatible incubator. Arch Med Sci 2016; 12: 1064-70.
9. Jiang A, Tian J, Li R, et al. Alterations of regional spontaneous brain activity and gray matter volume in the blind. Neural Plast 2015; 2015: 141950.

10. Pan WJ, Wu G, Li CX, Lin F, Sun J, Lei H. Progressive atrophy in the optic pathway and visual cortex of early blind Chinese adults: a voxel-based morphometry magnetic resonance imaging study. Neuroimage 2007; 37: 212-20.

11. Reislev NL, Kupers R, Siebner HR, Ptito M, Dyrby TB. Blindness alters the microstructure of the ventral but not the dorsal visual stream. Brain Struct Funct 2016; 221: 2891-903.

12. Chen HJ, Jiang LF, Sun T, Liu J, Chen QF, Shi HB. Resting-state functional connectivity abnormalities correlate with psychometric hepatic encephalopathy score in cirrhosis. Eur J Radiol 2015; 84: 2287-95.

13. Zhou C, Hu X, Hu J, et al. Altered brain network in amyotrophic lateral sclerosis: a resting graph theory-based network study at voxel-wise level. Front Neurosci 2016; 10: 204.

14. Zuo XN, Ehmke R, Mennes M, et al. Network centrality in the human functional connectome. Cereb Cortex 2012; 22: 1862-75.

15. Wu GR, Stramaglia S, Chen H, Liao W, Marinazzo D. Mapping the voxel-wise effective connectome in resting state FMRI. PLoS One 2013; 8: e73670.

16. Tomasi D, Volkow ND. Functional connectivity hubs in the human brain. Neuroimage 2011; 57: 908-17.

17. Di Martino A, Zuo XN, Kelly C, et al. Shared and distinct intrinsic functional network centrality in autism and attention-deficit/hyperactivity disorder. Biol Psychiatry 2013; 74: 623-32.

18. Lou Y, Huang P, Li D, et al. Altered brain network centrality in depressed Parkinson's disease patients. Mov Disord 2015; 30: 1777-84.

19. Alghamdi HF. Causes of irreversible unilateral or bilateral blindness in the Al Baha region of the Kingdom of Saudi Arabia. Saudi J Ophthalmol 2016; 30: 189-93.

20. Cai F, Gao L, Gong H, et al. Network centrality of resting-state $\mathrm{fMRI}$ in primary angle-closure glaucoma before and after surgery. PLoS One 2015; 10: e0141389.

21. Weliky M, Fiser J, Hunt RH, Waqner DN. Coding of natural scenes in primary visual cortex. Neuron 2003; 37: 703-18.

22. Chatterjee S, Callaway EM. Parallel colour-opponent pathways to primary visual cortex. Nature 2003; 426: 668-71.

23. Kelly SP, Gomez-Ramirez M, Foxe JJ. Spatial attention modulates initial afferent activity in human primary visual cortex. Cereb Cortex 2008; 18: 2629-36.

24. Shu N, Li J, Li K, Yu C, Jiang T. Abnormal diffusion of cerebral white matter in early blindness. Hum Brain Mapp 2009; 30: 220-7.

25. .Zhong YF, Tang ZH, Qiang JW, et al. Changes in DTI parameters in the optic tracts of macaque monkeys with monocular blindness. Neurosci Lett 2017; 636: 248-53.

26. Weaver KE, Richards TL, Saenz M, Petropoulos H, Fine I. Neurochemical changes within human early blind occipital cortex. Neuroscience 2013; 252: 222-33.

27. Qin W, Xuan Y, Liu Y, Jiang T, Yu C. Functional connectivity density in congenitally and late blind subjects. Cereb Cortex 2015; 25: 2507-16.

28. Zhong YF, Tang ZH, Qiang JW, et al. Changes in DTI parameters in the optic tracts of macaque monkeys with monocular blindness. Neurosci Lett 2017; 636: 248-53.

29. Sincich LC, Horton JC. The circuitry of V1 and V2: integration of color, form, and motion. Annu Rev Neurosci 2005; 28: 303-26. 
30. Cohen A, Buia C, Tiesinga P. Dependence of V2 illusory contour response on $\mathrm{V} 1$ cell properties and topographic organization. Biol Cybern 2014; 108: 337-54.

31. Thomas OM, Cumming BG, Parker AJ. A specialization for relative disparity in V2. Nat Neurosci 2002; 5: 472-8.

32. Poggio GF, Motter BC, Squatrito S, Trotter Y. Responses of neurons in visual cortex (V1 and V2) of the alert macaque to dynamic random-dot stereograms. Vision Res 1985; 25: 397-406.

33. Gonzalez F, Perez R. Neural mechanisms underlying stereoscopic vision. Prog Neurobiol 1998; 55: 191-224.

34. Vanni S, Tanskanen T, Seppä M, Uutela K, Hari R. Coinciding early activation of the human primary visual cortex and anteromedial cuneus. Proc Natl Acad Sci USA 2001; 98: 2776-80.

35. Baddeley R, Abbott LF, Booth MC, et al. Responses of neurons in primary and inferior temporal visual cortices to natural scenes. Proc Biol Sci 1997; 264: 1775-83.

36. McKee JL, Riesenhuber M, Miller EK, Freedman DJ. Task dependence of visual and category representations in prefrontal and inferior temporal cortices. J Neurosci 2014; 34: 16065-75.

37. Hamamé CM, Vidal JR, Ossandón T, et al. Reading the mind's eye: online detection of visuo-spatial working memory and visual imagery in the inferiortemporal lobe. Neuroimage 2012; 59: 872-9.

38. Yu C, Liu Y, Li J, et al. Altered functional connectivity of primary visual cortex in early blindness. Hum Brain Mapp 2008; 29: 533-43.

39. Seitz RJ, Nickel J, Azari NP. Functional modularity of the medial prefrontal cortex: involvement in human empathy. Neuropsychology 2006; 20: 743-51.

40. Harenski CL, Hamann S. Neural correlates of regulating negative emotions related to moral violations. Neuroimage 2006; 30: 313-24.

41. Gao B, Wang Y, Liu W, et al. Spontaneous activity associated with delusions of schizophrenia in the left medial superior frontal gyrus: a resting-state fMRI study. PLoS One 2015; 10: e0133766.

42. Myung W, Na KS, Ham BJ, Oh SJ, Ahn HW, Jung HY. Decreased medial frontal gyrus in patients with adjustment disorder. J Affect Disord 2016; 191: 36-40. 